\title{
A REMARK ON AN ALGEBRAIC RIEMANN-ROCH FORMULA FOR FLAT BUNDLES
}

\author{
HÉLÈNE ESNAULT
}

\section{INTRODUCTION}

Let $X$ be a smooth variety defined over an algebraically closed field $k$, and let $(E, \nabla)$ be a bundle with an integrable connection. Then $(E, \nabla)$ carries algebraic classes $c_{n}(E, \nabla)$ in the subgroup $\mathbb{H}^{n}\left(X, \Omega^{\infty} \mathcal{K}_{n}\right)$ of the group of algebraic differential characters $A D^{n}(X)$ consisting of the classes mapping to 0 in $H^{0}\left(X, \Omega^{2 n}\right)$. These classes lift the Chern classes $c_{n}(E, \nabla) \in C H^{n}(X)$, the algebraic Chern-Simons invariants $w_{n}(E, \nabla) \in H^{0}\left(X, \mathcal{H}_{D R}^{2 n-1}\right)$ for $n \geq 2$, as well as the analytic secondary invariants $c_{n}^{\text {an }}(E, \nabla) \in H^{2 n-1}\left(X_{\text {an }}, \mathbb{C} / \mathbb{Z}(n)\right)$ when $k=\mathbb{C}$ (see [5], [3], [6] and section 1).

Let now $f: X \rightarrow S$ be a smooth projective morphism. Then the de Rham cohomology sheaves $R^{j} f_{*}\left(\Omega_{X / S}^{\bullet} \otimes E, \nabla\right)$ carrie the (flat) GaußManin connection, still denoted by $\nabla$. Therefore one can ask for a Riemann-Roch formula relating

$$
c_{n}\left(\sum(-1)^{j}\left(R^{j} f_{*}\left(\Omega_{X / S}^{\bullet} \otimes E, \nabla\right), \nabla\right)\right)
$$

and $c_{m}(E, \nabla)$, as the classes $c_{n}(E, \nabla)$ verify the Whitney product formula for exact sequences of bundles with compatible (flat) connections. This formula should be compatible with Riemann-Roch-Grothendieck formula in $C H^{n}(S)$, and with Bismut-Lott and Bismut formula in $H^{2 n-1}\left(X_{\text {an }}, \mathbb{C} / \mathbb{Z}(n)\right)$ when $k=\mathbb{C}([1]$, [2] $)$. In this note, we propose an answer in the case $X=Y \times S$ and $f$ is the projection:

Theorem 0.0.1. Let $Y$ be a smooth projective variety of dimension d and $S$ be a smooth variety. Let $(E, \nabla)$ be a bundle with a flat connection on $X=Y \times S$. Then

$$
c_{n}\left(\sum_{j}(-1)^{j} R^{j} f_{*}\left(\Omega_{X / S}^{\bullet} \otimes E, \nabla\right), \nabla\right)=\left.(-1)^{d} f_{*} c_{n}(E, \nabla)\right|_{c_{d}\left(\Omega_{X / S}^{1}\right)}
$$

This work has been partly supported by the DFG Forschergruppe "Arithmetik und Geometrie". 
where $f$ is the projection to $S$, where the right hand side means that one takes a zero cycle $\Sigma=\sum_{i} m_{i} p_{i} \subset Y$ representing $c_{d}\left(\Omega_{Y}^{1}\right)$, and

$$
\left.(-1)^{d} f_{*} c_{n}(E, \nabla)\right|_{c_{d}\left(\Omega_{X / S}^{1}\right)}=\left.\sum_{i} m_{i} c_{n}(E, \nabla)\right|_{\left\{p_{i}\right\} \times S} \in \mathbb{H}^{n}\left(S, \Omega^{\infty} \mathcal{K}_{n}\right)
$$

(see section 1).

For $n=1, \mathbb{H}^{1}\left(S, \Omega^{\infty} \mathcal{K}_{1}\right)$ is the group of isomorphism class of rank one bundles with an integrable connection. Thus the formula for the determinant bundle as a flat bundle is similar to Deligne-Laumon formula for the determinant bundle of a $\ell$-adic sheaf $E$ over $X$ with $S=$ Spec $\overline{\mathbb{F}}_{p}$, $(p, \ell)=1([4,[7])$. But here, we treat only the case where $f$ is split, which does not occur in the $\ell$-adic content. However, we don't know what would be a corresponding formula in higher codimension in the arithmetic case.

The method used is inspired by the work of Hitchin and Simpson. We deform

$$
\sum_{j}(-1)^{j}\left(R^{j} f_{*}\left(\Omega_{X / S}^{\bullet} \otimes E, \nabla\right), \nabla\right)
$$

to the alternate sum of a flat structure

$$
\left(R^{j} f_{*}\left(\Omega_{X / S}^{\bullet} \otimes E, \alpha\right), \nabla_{i}\right)
$$

defined on the cohomology of a Higgs bundle, where the Higgs structure is defined by a form $\alpha \in H^{0}\left(Y, \Omega_{Y}^{1}\right)$, if $H^{0}\left(Y, \Omega_{Y}^{1}\right) \neq 0$. If not, one has to introduce poles (see section 2).

0.1. Acknowledgements: I started discussing on possible RiemannRoch formulae with Spencer Bloch. The motivation coming from [四], [7] is due to him. I thank him for his generosity. I thank Gérard Laumon and Domingo Toledo for explaining me parts of their work. Finally I thank Eckart Viehweg for discussions on the use of logarithmic forms and for his encouragements.

\section{Trace OF Algebraic DifFerential CHARACTERS}

1.1. Algebraic differential characters. Let $X$ be a smooth variety, $D \subset X$ be a normal crossing divisor, $\mathcal{K}_{n}$ be the Zarisky sheaf image of the Zarisky sheaf of Milnor $K$-theory in $K_{n}^{M}(k(x))$. We recall [6], section 2 , that the group

$$
A D^{n}(X, D):=\mathbb{H}^{n}\left(X, \mathcal{K}_{n} \stackrel{d \log }{\longrightarrow} \Omega_{X}^{n}(\log D) \rightarrow \ldots \rightarrow \Omega_{X}^{2 n-1}(\log D)\right)
$$

has a commutative product

$$
A D^{m}(X, D) \times A D^{n}(X, D) \rightarrow A D^{m+n}(X, D)
$$


respecting the subgroup

$$
\begin{aligned}
& \mathbb{H}^{n}\left(X, \Omega_{X}^{\infty}(\log D) \mathcal{K}_{n}\right):= \\
& \mathbb{H}^{n}\left(X, \mathcal{K}_{n} \stackrel{d \log }{\longrightarrow} \Omega_{X}^{n}(\log D) \rightarrow \ldots\right) \\
& \quad=\operatorname{Ker}\left(A D^{n}(X, D) \rightarrow H^{0}\left(X, \Omega_{X}^{2 n}(\log D)\right)\right.
\end{aligned}
$$

and compatible with the products in $\mathcal{K}_{n}$ and $\Omega_{X}^{\geq n}(\log D)$. A bundle $(E, \nabla)$ with a $\Omega_{X}^{1}(\log D)$ connection has functorial and additive classes $c_{n}(E, \nabla) \in A D^{n}(X, D)$, lying in $\mathbb{H}^{n}\left(X, \Omega_{X}^{\infty}(\log D) \mathcal{K}_{n}\right)$ when $\nabla^{2}=0$.

Lemma 1.1.1. Let $X=\mathbb{P}^{1} \times S$, with $S$ smooth, $B \subset \mathbb{P}^{1}$ be a divisor, $D=B \times S, p_{i}, i=1,2$ be the projections of $X$ to $\mathbb{P}^{1}$ and $S$. Then one has a direct sum decomposition

$$
\begin{aligned}
& p_{2}^{*} \oplus p_{2}^{*} \otimes p_{1}^{*}: \\
& \begin{aligned}
\mathbb{H}^{n}\left(S, \Omega_{S}^{\infty} \mathcal{K}_{n}\right) \oplus \mathbb{H}^{n-1}\left(S, \Omega_{S}^{\infty} \mathcal{K}_{n-1}\right) \otimes & \mathbb{H}^{1}\left(\mathbb{P}^{1}, \Omega_{\mathbb{P}^{1}}^{\infty}(\log B) \mathcal{K}_{1}\right) \\
& \rightarrow \mathbb{H}^{n}\left(X, \Omega_{X}^{\infty}(\log D) \mathcal{K}_{n}\right) .
\end{aligned}
\end{aligned}
$$

Proof. One has the Künneth formulae

$$
\begin{aligned}
& H^{\ell}\left(X, \mathcal{K}_{n}\right)= \\
& p_{2}^{*} H^{\ell}\left(S, \mathcal{K}_{n}\right) \oplus p_{2}^{*} H^{\ell-1}\left(S, \mathcal{K}_{n-1}\right) \cup p_{1}^{*} H^{1}\left(\mathbb{P}^{1}, \mathcal{K}_{1}\right) \\
& \mathbb{H}^{\ell}\left(X, \Omega_{\bar{X}}^{\geq n}(\log D)\right)= \\
& p_{2}^{*} \mathbb{H}^{\ell}\left(S, \Omega_{\bar{S}}^{\geq n}\right) \oplus p_{2}^{*} \mathbb{H}^{\ell-1}\left(S, \Omega_{\bar{S}}^{\geq n-1}\right) \cup p_{1}^{*} \mathbb{H}^{0}\left(\mathbb{P}^{1}, \Omega_{\mathbb{P}^{1}}^{1}(\log B)\right) \\
& \text { if } B \neq \phi \\
& \mathbb{H}^{\ell}\left(X, \Omega_{\bar{X}}^{\geq n}(\log D)\right)= \\
& =p_{2}^{*} \mathbb{H}^{\ell}\left(S, \Omega_{\bar{S}}^{\geq n}\right) \oplus p_{2}^{*} \mathbb{H}^{\ell-2}\left(S, \Omega_{\bar{S}}^{\geq n-1}\right) \cup p_{1}^{*} H^{0}\left(\mathbb{P}^{1}, \Omega_{\mathbb{P}^{1}}^{1}\right) \\
& \text { if } B=\phi .
\end{aligned}
$$

Moreover, in the long exact cohomology sequence associated to the short exact sequence

$$
0 \rightarrow \Omega_{X}^{\geq n}(\log D)[n-1] \rightarrow \Omega_{X}^{\infty}(\log D) \mathcal{K}_{n} \rightarrow \mathcal{K}_{n} \rightarrow 0
$$

the map

$$
H^{\ell}\left(X, \mathcal{K}_{n}\right) \stackrel{d \log }{\longrightarrow} \mathbb{H}^{\ell+n}\left(X, \Omega_{\bar{X}}^{\geq n}(\log D)\right)
$$

respects this direct sum composition. (If $B \neq \phi$, the term

$$
p_{2}^{*} H^{\ell-1}\left(S, \mathcal{K}_{n-1}\right) \cup p_{1}^{*} H^{1}\left(\mathbb{P}^{1}, \mathcal{K}_{1}\right)
$$

maps to zero). This shows the lemma. 


\subsection{Trace.}

Proposition 1.2.1. Let $X=Y \times S$, with $Y$ smooth projective, $S$ smooth, and $f: X \rightarrow S$ be the projection. Let $\Sigma=\sum m_{i} p_{i}$ be a zero cycle in $Y$, and $(E, \nabla)$ be a bundle with an integrable connection on $X$. Then

$$
\left.f_{*} c_{n}(E, \nabla)\right|_{\Sigma \times S}:=\sum m_{i} c_{n}\left(\left.(E, \nabla)\right|_{\left\{p_{i}\right\} \times S}\right) \in \mathbb{H}^{n}\left(S, \Omega_{S}^{\infty} \mathcal{K}_{n}\right)
$$

does not depend on the choice of the representative $\Sigma$ in its equivalence class $[\Sigma] \in C H_{0}(S)$.

Proof. Let $\Sigma^{\prime}=\sum m_{i} p_{i}^{\prime}$ be another choice. Then there are rational functions $f_{i}$ on curves $C_{i} \subset Y$ such that $\Sigma-\Sigma^{\prime}=\sum \operatorname{div} f_{i}$. Therefore it is sufficient to prove the following: let $\nu: C \rightarrow Y$ be the normalization of an irreducible curve $\nu(C) \subset X$, and $\varphi: C \rightarrow \mathbb{P}^{1}$ be a non trivial rational function on $C$. Then

$$
c_{n}\left(\left.\left(\nu \times \operatorname{id}_{S}\right)^{*}(E, \nabla)\right|_{\varphi^{-1}(0) \times S}\right)=c_{n}\left(\left.\left(\nu \times \operatorname{id}_{S}\right)^{*}(E, \nabla)\right|_{\varphi^{-1}(\infty) \times S}\right) .
$$

Let $(\mathcal{E}, D):=\left(\nu \times \operatorname{id}_{X}\right)^{*}(E, \nabla)$. Let $B \subset \mathbb{P}^{1}$ be the ramification locus of $\varphi, \pi=\varphi \times \operatorname{id}_{S}: C \times S \rightarrow \mathbb{P}^{1} \times S$. Then $\pi_{*}(\mathcal{E}, D)$ is a bundle with an integrable connection with logarithmic poles along $D=B \times S$, and has classes

$$
c_{n}\left(\pi_{*}(\mathcal{E}, D)\right) \in \mathbb{H}^{n}\left(\mathbb{P}^{1} \times S, \Omega_{X}^{\infty}(\log D) \mathcal{K}_{n}\right) .
$$

For $t \notin B$, then

$$
c_{n}\left(\left.\pi_{*}(\mathcal{E}, D)\right|_{\{t\} \times S}\right)=\sum_{s \in \varphi^{-1}(t)} c_{n}\left(\left.(E, D)\right|_{\{s\} \times S}\right)=\left.c_{n}(\mathcal{E}, D)\right|_{\varphi^{-1}(t) \times S} .
$$

For $t \in B$, the residue map

$$
\operatorname{res}_{\{t\} \times S}: p_{2}^{*} \Omega_{S}^{n-1} \otimes p_{1}^{*} \Omega_{\mathbb{P}^{1}}^{1}(\log B) \rightarrow \Omega_{\{t\} \times S}^{n-1}
$$

verifies

$$
\operatorname{res}_{\{t\} \times S} \otimes_{\mathcal{O}_{\mathbb{P}^{1} \times S}} \mathcal{O}_{\{t\} \times S}=\text { Identity }_{\{t\} \times S} .
$$

Therefore there is a canonical splitting

$$
\left.\Omega_{X}^{n}(\log D)\right|_{\{t\} \times S}=\Omega_{S}^{n} \oplus \Omega_{\{t\} \times S}^{n-1}
$$

Thus the connection

$$
\pi_{*} D: \pi_{*} \mathcal{E} \rightarrow \Omega_{\mathbb{P}^{1} \times S}^{1}(\log D) \otimes \pi_{*} \mathcal{E}
$$

restricts to

$$
\left.\pi_{*} D\right|_{\{t\} \times S}:\left.\left.\pi_{*} \mathcal{E}\right|_{\{t\} \times S} \rightarrow\left(\Omega_{S}^{1} \oplus \mathcal{O}_{S}\right) \otimes \pi_{*} \mathcal{E}\right|_{\{t\} \times S}
$$

defining by projection the connection

$$
\left.\overline{\pi_{*} D}\right|_{\{t\} \times S}:\left.\left.\pi_{*} \mathcal{E}\right|_{\{t\} \times S} \rightarrow \Omega_{S}^{1} \otimes\left(\pi_{*} \mathcal{E}\right)\right|_{\{t\} \times S} .
$$


The integrability of $\left.\pi_{*} D\right|_{\{t\} \times S}$ implies the integrability of the genuine connection $\left.\overline{\pi_{*} D}\right|_{\{t\} \times S}$, and

$$
c_{n}\left((\mathcal{E}, D) \mid \varphi^{-1}(t) \times S\right)=c_{n}\left(\left.\pi_{*} \mathcal{E}\right|_{\{t\} \times S},\left.\overline{\pi_{*} D}\right|_{\{t\} \times S}\right) .
$$

Now by [7],

$$
c_{n}\left(\pi_{*}(\mathcal{E}, D)\right)=p_{2}^{*} a+\sum_{i} p_{2}^{*} b_{i} \cup p_{1}^{*} c_{i}
$$

where $a \in \mathbb{H}^{n}\left(S, \Omega_{S}^{\infty} \mathcal{K}_{n}\right)$, and the preceding discussion shows that

$$
c_{n}\left(\left.(\mathcal{E}, D)\right|_{\varphi^{-1}(t) \times S}\right)=a \in \mathbb{H}^{n}\left(S, \Omega_{S}^{\infty} \mathcal{K}_{n}\right)
$$

whether $t \in B$ or $t \notin B$.

\section{Proof of THE THEOREM}

2.1. Notations. Let $H_{0}, \ldots, H_{N}$ be restriction of the coordinate hyperplanes $H_{0}^{\prime}, \ldots, H_{N}^{\prime}$ in an embedding $Y \subset \mathbb{P}^{N}$. Since $\Omega_{\mathbb{P}^{N}}^{1}\left(\log H_{0}^{\prime} \cup\right.$ $\left.\ldots H_{N}^{\prime}\right) \cong \oplus_{1}^{N} \mathcal{O}_{\mathbb{P}^{N}}$, the sheaf $\Omega_{Y}^{1}\left(\log H_{0} \cup \ldots H_{N}\right)$ is globally generated. We denote by $H^{(\ell)}$ the normalization of the $\ell$ by $\ell$ intersections of the $H_{j}, H^{(0)}=X, H^{(\delta)}=\emptyset$ for $\delta>d$, by $H$ the union of the $H_{j}$, by

$$
\Omega_{H^{(\ell)}}^{a}\left(\log H^{(\ell+1)}\right)
$$

the sheaf of a form on $H^{(\ell)}$ with logarithmic poles along the $(\ell+1)$ by $(\ell+1)$ intersections. One has the following resolution of the de Rham complex

$$
\begin{gathered}
\Omega_{X / S}^{\bullet} \rightarrow \Omega_{X / S}^{\bullet}(\log (H \times S)) \rightarrow \Omega_{H^{(1)} \times S / S}^{\bullet-1}\left(\log \left(H^{(2)} \times S\right)\right) \rightarrow \ldots \\
\rightarrow \Omega_{H^{(\ell)} \times S / S}^{\bullet-\ell}\left(\log \left(H^{(\ell+1)} \times S\right)\right) \rightarrow \ldots \\
\rightarrow \Omega_{H^{(d)} \times S / S}^{\bullet-d} \rightarrow 0 .
\end{gathered}
$$

2.2. Proof. The resolution 2.1.1 is compatible with the Gauß-Manin connection, as

$$
H^{(\ell)} \times S
$$

is dominant over $S$. 
Therefore in the $K$ group $K$ (S, flat) of bundles on $S$ with an integrable connection, one has

$$
\begin{aligned}
& \sum_{j}(-1)^{j} R^{j} f_{*}\left(\Omega_{X / S}^{\bullet} \otimes E, \nabla\right) \\
&=\sum(-1)^{j} R^{j} f_{*}\left(\Omega_{X / S}^{\bullet}(\log H \times S) \otimes E, \nabla\right) \\
&+\sum(-1)^{j} R^{j} f_{*}\left(\Omega_{H^{(1)} \times S / S}^{\bullet}\left(\log \left(H^{(2)} \times S\right)\right) \otimes E, \nabla\right) \\
&+\ldots+\sum(-1)^{j} R^{j} f_{*}\left(\Omega_{H^{(\ell)} \times S / S}^{\bullet}\left(\log \left(H^{(\ell)} \times S\right)\right) \otimes E, \nabla\right) \\
&+\ldots+f_{*}\left(\Omega_{H^{(d)} \times S / S}^{\bullet} \otimes E, \nabla\right) .
\end{aligned}
$$

On the other hand

$$
\begin{aligned}
& c_{d}\left(\Omega_{X / S}^{\bullet}\right)= c_{d}\left(\Omega_{X / S}^{1}(\log H)\right) \\
& \quad-c_{d-1}\left(\Omega_{H^{\ell} \times S / S}^{1}\left(\log \left(H^{(2)} \times S\right)\right)\right. \\
&+\ldots+(-1)^{\ell} c_{d-\ell}\left(\Omega_{H^{(\ell)} \times S / S}^{1}\left(\log \left(H^{(\ell+1)} \times S\right)\right)\right. \\
& \quad+\ldots+(-1)^{d}\left[H^{(d)} \times S\right]
\end{aligned}
$$

where $\left[H^{(d)} \times S\right]$ means the codimension $d$ cycle, image of $H^{(d)} \times S$ in $Y \times S$.

Therefore, one just has to prove the following formula

$$
\begin{aligned}
c_{n}\left(\sum ( - 1 ) ^ { j } \left(R ^ { j } f _ { * } \left(\Omega_{X / S}^{\bullet}(\log D\right.\right.\right. & \times S) \otimes E), \nabla), \nabla)) \\
& \left.=\left.(-1)^{d} f_{*} c_{n}(E, \nabla)\right|_{c_{d}\left(\Omega_{X / S}^{1}(\log (H \times S))\right.}\right) .
\end{aligned}
$$

We denote by $\tau: \Omega_{X}^{1}(\log (H \times S)) \rightarrow f^{*} \Omega_{S}^{1}$ the splitting of the one forms, which induces a splitting

$$
\tau: \Omega_{X}^{\bullet}(\log (H \times S)) \rightarrow f^{*} \Omega_{S}^{\bullet}
$$

of the de Rham complex, where the differential $f^{*} \Omega_{S}^{i} \rightarrow f^{*} \Omega_{S}^{i+1}$ is defined by $\tau d \iota, \iota: f^{*} \Omega_{S}^{i} \rightarrow \Omega_{X}^{i}(\log (H \times S))$ being the natural embedding. This defines a $f^{*} \Omega_{S}^{1}$ valued connection $\nabla_{\tau}$ on $\Omega_{X / S}^{i}(\log (H \times S)) \otimes E$ by embedding $\Omega_{X / S}^{i}(\log (H \times S)) \otimes E$ into $\Omega_{X}^{i}(\log (H \times S)) \otimes E$ via the splitting, then taking $\nabla$, then projecting onto the factor

$$
f^{*} \Omega_{S}^{1} \otimes \Omega_{X / S}^{1}(\log (H \times S)) \otimes E
$$

with the sign $(-1)^{i}$. The integrability condition $\nabla^{2}=0$ then implies that

$$
\nabla_{\tau} \circ \nabla_{X / S}=\nabla_{X / S} \circ \nabla_{\tau}
$$


where

$$
\nabla_{X / S}: \Omega_{X / S}^{i}(\log (H \times S)) \otimes E \rightarrow \Omega_{X / S}^{i+1}(\log (H \times S)) \otimes E .
$$

Taking cohomology defines a connection, still denoted by $\nabla_{\tau}$ $\nabla_{\tau}: R^{j} f_{*} \Omega_{X / S}^{i}(\log (H \times S)) \otimes E \rightarrow \Omega_{S}^{1} \otimes R^{j} f_{*} \Omega_{X / S}^{i}(\log (H \times S) \otimes E$.

The integrability of $\nabla$ implies the integrability of $\nabla_{\tau}$. Therefore in $K(S$, flat) one has

$$
\begin{aligned}
\left(R ^ { j } \left(f _ { * } \left(\Omega_{X / S}^{\bullet}(\log (H\right.\right.\right. & \times S)) \otimes E, \nabla), \nabla) \\
& \left.=\oplus_{i} R^{j-i}\left(f_{*} \Omega_{X / S}^{i}(\log (H \times S)) \otimes E, \nabla_{\tau}\right), \nabla_{\tau}\right)
\end{aligned}
$$

and

$$
\begin{aligned}
\sum_{j}(-1)^{j}\left(R^{j} f_{*} \Omega_{X / S}^{\bullet}\right. & (\log (H \times S) \otimes E, \nabla), \nabla) \\
= & \sum_{i, j}(-1)^{i+j}\left(R^{j} f_{*} \Omega_{X / S}^{i}(\log (H \times S)) \otimes E, \nabla_{\tau}\right) .
\end{aligned}
$$

Let $\alpha \in H^{0}\left(Y, \Omega_{Y}^{1}(\log H)\right)$ be a non-trivial generic section. We still denote by $\alpha$ the corresponding form $p_{1}^{*} \alpha \in H^{0}\left(X, \Omega_{X / S}^{1}(\log (H \times S))\right)$. Then, it defines a morphism

$$
\alpha_{X / S}: \Omega_{X / S}^{i}(\log (H \times S)) \otimes E \rightarrow \Omega_{X / S}^{i+1}(\log (H \times S)) \otimes E
$$

by $\alpha_{X / S}(w \otimes e)=\alpha \wedge w \otimes e$. As $d \alpha=0$, one has

$$
\alpha_{X / S} \circ \nabla_{\tau}=\nabla_{\tau} \circ \alpha_{X / S}
$$

Thus in $K$ ( $S$, flat) one has

$$
\begin{aligned}
& \sum_{i, j}(-1)^{i+j}\left(R^{j} f_{*}\left(\Omega_{X / S}^{i}(\log (H \times S)) \otimes E, \nabla_{\tau}\right), \nabla_{\tau}\right) \\
&=\sum_{j}(-1)^{j}\left(R^{j} f_{*}\left(\Omega_{X / S}^{\bullet}(\log (H \times S)) \otimes E, \alpha_{X / S}\right), \nabla_{\tau}\right) .
\end{aligned}
$$

On the other hand, the complex

$$
\left(\Omega_{X / S}^{\bullet}(\log (H \times S)), \alpha_{X / S}\right)
$$

is quasi-isomorphic to $\mathcal{O}_{\Sigma \times S}[-d]$, where $\Sigma$ is the zero set of $\alpha$, and one furthermore has a commutative diagram of complexes

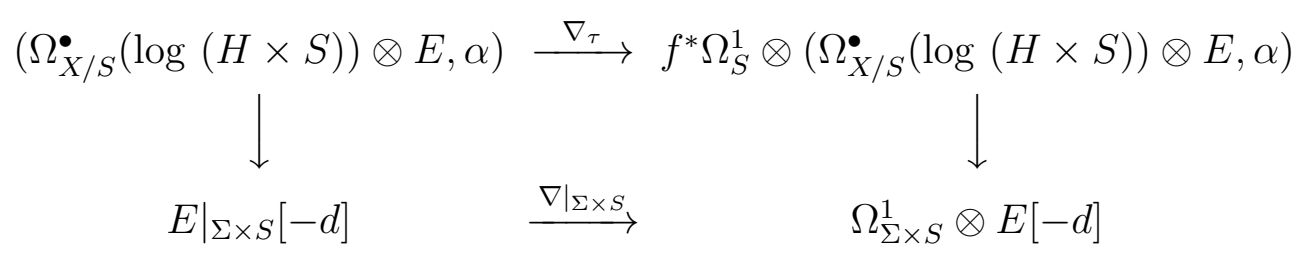


This shows the equality in $K$ ( $S$, flat)

$$
\begin{aligned}
\sum_{j}(-1)^{j}\left(R ^ { j } f _ { * } \left(\Omega_{X / S}^{\bullet}(\log (H \times S) \otimes E, \alpha)\right.\right. & \left., \nabla_{\tau}\right) \\
& =\left.(-1)^{d} \oplus_{\sigma \in \Sigma}(E, \nabla)\right|_{\sigma \times S}
\end{aligned}
$$

and finishes the proof.

\section{REFERENCES}

[1] Bismut, J.-M.: Eta invariants, differential characters and flat vector bundles, Preprint 1995.

[2] Bismut, J.-M., Lott, J.: Flat vector bundles, direct images and higher real analytic torsion, J. of the AMS 8 (2) (1995), 291-363.

[3] Bloch, S.; Esnault, H.: Algebraic Chern-Simons theory, to appear in American J. of Mathematics.

[4] Deligne, P.: Les constantes des équations fonctionnelles des fonctions $L, \mathrm{LN}$ 349 (1973), 55-106.

[5] Esnault, H.: Characteristic class of flat bundles, II. $K$-Theory 6 (1992), 45-56.

[6] Esnault, H.: Algebraic Differential Characters, Preprint 1996, 27 pages.

[7] Laumon, G.: Transformation de Fourier, constantes d' équations fonctionnelles et conjecture de Weil, Publ. Math. IHES 65 (1987), 131-210.

Universität Essen, FB6 Mathematik, 45117 Essen, Germany

E-mail address: esnault@uni-essen.de 